\title{
Vivências do cotidiano hospitalar no processo formativo da residência em saúde: estratégias pedagógicas e desafios para a prática
}

\section{Experiences of hospital everyday in the formative process of health residence: pedagogical strategies and challenges for practice}

\author{
Ana Maria Araújo Salomão' • Bruna Pereira de Andrade ${ }^{2} \bullet$ Leonardo Nunes Ferreira $^{3}$ \\ José Adelmo da Silva Filho ${ }^{4}$ Cleide Carneiro $^{5} \bullet$ Heraldo Simões Ferreira $^{6} \bullet$ Antonio Germane Alves Pinto ${ }^{7}$
}

\begin{abstract}
RESUMO
Objetivo: compreender o processo de formação interdisciplinar em um programa de residência multiprofissional com ênfase hospitalar. Método: estudo com abordagem qualitativa, enfoque crítico- reflexivo e ancorado na formação para integralidade em saúde. $O$ cenário da pesquisa foi um hospital de referência terciária, em Fortaleza-Ceará, que contém a residência multiprofissional voltada ao cuidado cardiopulmonar, campo que comporta a população em estudo do presente trabalho, sendo 10 entrevistados, entre residentes e preceptores que orientam o processo de trabalho das categorias profissionais. A coleta de dados foi dividida em duas fases: a priori aplicou-se a entrevista semiestruturada aos residentes e preceptores, posteriormente, realizou-se a análise documental do Projeto Político Pedagógico da Residência Integrada em Saúde. Resultados: os residentes reconhecem a importância do programa para o aperfeiçoamento profissional, para o fortalecimento da gestão em saúde e para a construção de uma formação pautada no trabalho multiprofissional, e com um método problematizador, coerente com as necessidades da comunidade. Evidenciam-se vulnerabilidades, enfatizadas pela pouca valorização dos profissionais e por métodos formativos ainda distantes da prática cotidiana. Conclusão:A residência como processo formativo na relação entre ensino-serviço, no qual ocupa espaço nos cenários de saúde, buscando alinhamento nas propostas do Sistema Único de Saúde e no fazer saúde.
\end{abstract}

Palavras-chave: Práticas Interdisciplinares; Equipe Multiprofissional; Hospitais de Ensino.

\begin{abstract}
Objective: understand or process of interdisciplinary training in a multiprofessional residency program with hospital phase. Method: study with a qualitative approach, with a critical-reflexive focus. The research scenario was a tertiary referral hospital, in Fortaleza-Ceará, which contains the multiprofessional residency focused on cardiopulmonary care, a field that encompasses the population under study in the present study, 10 of whom were interviewed, including residents and preceptors who guide the process of professional categories. Data collection was divided into two phases: a priori the semi-structured interview was applied to residents and preceptors, afterwards, the documentary analysis of the Pedagogical Political Project of the Integrated Health Residence was carried out. Results: residents recognize the importance of the program for professional development, for strengthening health management and for building training based on multiprofessional work, and with a problematic method, consistent with the needs of the community.Vulnerabilities are evidenced, emphasized by the low valuation of professionals and by training methods that are still far from daily practice. Conclusion: Residence as a formative process in the relationship between teaching and service, in which it occupies space in health settings, seeking alignment in the proposals of the Unified Health System and in doing health.
\end{abstract}

Keywords: Interdisciplinary Practices; Multiprofessional Team; Teaching Hospitals.

NOTA

I Mestrado em Ensino na Saúde (UECE). Esp. em Farmacologia Clínica e Atenção Farmacêutica (IPOG). E-mail: anasalomaoaraujo3@gmail.com

2 Discente do curso de Enfermagem da Universidade Regional do Cariri. Bolsista do Programa de Educação pelo trabalho - PET/Saúde-Interprofissionalidade. Membro do Grupo de Pesquisa Clínica, Cuidado e Gestão em Saúde (GPCLIN/URCA/CNPq). E-mail: brunaandrade888@gmail.com

3 Discente do curso de Medicina da Faculdade Estácio de Juazeiro do Norte. Membro do Grupo de Pesquisa Clínica, Cuidado e Gestão em Saúde (GPCLIN/URCA/ CNPq).E-mail: leonardofn88@gmail.com

4 Mestrando em Enfermagem (URCA). Esp. em Saúde Mental Coletiva (ESPCE). Membro do Grupo de Pesquisa Clínica, Cuidado e Gestão em Saúde (GPCLIN/ URCA/CNPq). E-mail: adelmofl2@gmail.com

5 Doutorado em Serviço Social (UNESP). Professora adjunta da Universidade Estadual do Ceará. Docente do Curso de Mestrado Profissional Ensino na Saúde (UECE). E-mail: cleide.carneiro@uece.br

6 Doutorado em Saúde Coletiva (UECE). Professor Adjunto da Universidade Estadual do Ceará. Docente do Curso de Mestrado Profissional Ensino na Saúde (UECE). Líder do Grupo de Estudos e Pesquisa em Educação Física Escolar (GEPEFE/UECE/CNPq). E-mail: heraldo.simoes@uece.br

7 Doutorado em Saúde Coletiva (UECE). Docente do Curso de Mestrado Profissional Ensino na Saúde (UECE). Professor Adjunto da Universidade Regional do Cariri. Líder do Grupo de Pesquisa Clínica, Cuidado e Gestão em Saúde (GPCLIN/URCA/CNPq). E-mail: germanepinto@hotmail.com 


\section{INTRODUÇÃO}

$\mathrm{Na}$ atual conjuntura da saúde, a inserção dos profissionais nos cenários de práticas propiciadas pelos programas de Residências Multiprofissionais em Saúde (RMS) vem sendo discutida e reconhecida, diante do novo panorama de formar profissionais com competências e habilidades necessárias para o cuidado integral da saúde do sujeito.

A necessidade de formar profissionais preocupados com a realidade local e com o social é mais premente devido ao perfil dos profissionais da área da saúde que estão sendo formados no modelo de ensino predominantemente fragmentado à luz das especialidades, visando as orientações clínicas, com práticas biologicistas, mecanicistas e individualizantes ${ }^{(1)}$.

As capacitações e programas de pós-graduação na área da saúde contribuem eficientemente para o aperfeiçoamento e mudanças na prática profissional(2). A presença contínua dos profissionais nos locais de produção das ações, como a oportunidade estratégica de aprendizagem coletiva e em equipe multiprofissional, contribui para esse entendimento e melhora as relações de poder nas práticas de saúde.

A RMS é uma modalidade de vivência e formação que tem como base a aprendizagem em serviço, caracterizada pela diferenciação no aprender, tendo em vista as atividades desenvolvidas pelo residente, que tem a oportunidade de adquirir experiência prática em diferentes cenários, gerando uma progressão dos conhecimentos e das aptidões técnicas. Permite, dessa forma, que o residente tenha o potencial de unir a interdisciplinaridade e os diversos saberes e fazeres no espaço de formação em saúde ${ }^{(3)}$.

A percepção dos estudantes sobre conhecer a realidade do sistema público é relevante, partindo do pressuposto que, ao vivenciar do Sistema Único de Saúde- SUS ainda na sua graduação, oportuniza identificar as fragilidades do sistema, o funcionamento e processo de trabalho, além da possibilidade de uma futura atuação neste cenário de prática ${ }^{(4)}$.

A proposta das residências em saúde multiprofissional objetiva formar profissionais para uma atuação mais incisiva no SUS. Destarte, as RMS que se apresentam no cenário brasileiro como uma perspectiva teórico-pedagógica convergente com os princípios e diretrizes da integralidade da atenção e da intersetorialidade do SUS, promovem o contato entre o trabalho e as práticas formativas, possibilitando mudanças no modelo tecnoassistencial a partir da atuação interprofissional(5).

O processo de formação da residência integrada em saúde com enfoque para a atuação multidisciplinar no processo de ensino-serviço é um desafio para a área. Intenciona-se com este estudo contribuir para o debate e aprofundamento sobre as residências multiprofissionais em saúde, e que se reflita sobre a formação dos profissionais de saúde no Brasil.

Diante do contexto, objetiva-se compreender o processo de formação interdisciplinar em um programa de residência multiprofissional com ênfase hospitalar

\section{MÉTODO}

Estudo com abordagem qualitativa, enfoque crítico -reflexivo e ancorado na formação para integralidade em saúde. Nesta abordagem, conformam-se as investigações de grupos, relações e análises de discursos e documentos $^{6}$. A ênfase na formação multiprofissional é um pressuposto ético, político e filosófico do processo ensino -aprendizagem na Educação Permanente em Saúde ${ }^{(5)}$.

O cenário da pesquisa foi um hospital de referência terciária em Fortaleza-Ceará. Conhecido como Centro e Referência de Alta Complexidade, que se dedica ao diagnóstico e tratamento de doenças cardiopulmonares, é responsável pelo serviço que mais efetua transplantes cardíacos por milhão de habitantes, destacando-se no transplante cardíaco de adultos e crianças.

O cenário de pesquisa é considerado um hospital escola, que atua como centro de ensino/ pesquisa e integra a Rede Nacional de Pesquisa Clínica em Hospitais de Ensino. Compõe ainda a Rede de Hospitais Sentinela da Agência Nacional de Vigilância Sanitária (ANVISA).

A Residência Multiprofissional está inserida em escola vinculada ao SUS do estado do Ceará, que acompanha a rede de atenção especializada e hospitalar deste estado. Este centro de ensino submeteu a proposta de uma residência multiprofissional voltada para o cuidado cardiopulmonar, cujo programa trabalha com 9 profissões, fundamentado na teoria de campo e núcleo.

No período da coleta, o programa de residência contava com 17 residentes, sendo 2 farmacêuticos, 2 cirurgiões dentistas, 3 psicólogos, 3 enfermeiros, I terapeuta ocupacional, 2 fisioterapeutas, 2 nutricionistas e 2 assistentes sociais. A definição da amostra dos residentes se deu por saturação teórica.

Obteve-se uma amostra representada por 10 sujeitos, que equivale a $50 \%$ dos residentes em exercício e $50 \%$ dos preceptores. Como critérios de inclusão, os residentes deveriam estar regularmente incorporados à $2^{\mathrm{a}}$ turma da Residência Integrada em Saúde (RIS), e que os preceptores atuassem nessa função há pelo menos I ano, e no caso de estarem desligados, que $\circ$ afastamento não tenha ocorrido há mais de um ano.

Realizou-se a investigação em campo para coleta de dados em dois momentos: análise documental pelo Projeto Político Pedagógico (PPP) da RIS e aplicação da entrevista semiestruturada com preceptores e residentes. Neste tipo de entrevista, o participante tem a possibi- 
lidade de discorrer sobre o tema em questão sem se prender à indagação formulada.

A fase da análise documental contribuiu para identificar se as diretrizes do PPP da RIS estão relacionadas com a integração multiprofissional e articulação teórico-prática da instituição.

Os dados foram organizados e avaliados por meio da análise temática de conteúdo. Essa análise compreende a pré-análise, exploração do material, tratamento dos resultados obtidos e interpretação. $\mathrm{A}$ análise temática trabalha com partes do texto como, por exemplo, uma palavra, uma frase, um tema, depois define as regras de contagem e depois classifica e agrega os dados ${ }^{(6)}$.

Ademais, confrontaram-se as falas dos respondentes com as ideias de autores que se dedicam às práxis teórica do objeto de estudo da pesquisa, com os resultados de estudos similares e com a própria opinião da autora, inserindo-se no estudo como pesquisadora.

O estudo foi submetido ao Comitê de Ética em Pesquisa (CEP) do Hospital de Messejana Dr. Carlos Alberto Studart Gomes, obtendo parecer favorável com número 2.05I.486 e CAEE 567805।6.0.300I.5039, conforme previsto pela resolução 466/I 2 do Conselho Nacional de Saúde do Ministério da Saúde, que regulamenta a pesquisa com seres humanos ${ }^{(7)}$.

Vale ressaltar que os entrevistados foram mantidos em anonimato, uma vez que suas falas não os identificam. Com isso, foi estabelecido que as falas destacadas ao longo do corpo do texto fossem denominadas como "depoimento de preceptor e de residente".

\section{RESULTADOS E DISCUSSÃO}

\section{Contexto de formação multiprofissional em saúde}

Deve ser parte da formação acadêmica a adoção de métodos ativos que unem o saber teórico, construído e aprimorado ao longo de anos de existência humana, ao cenário prático, valorizando os saberes e vivências apresentadas por cada integrante. Neste contexto, a RMS valida-se como prática oportuna na integração de diferentes profissionais, possibilitando o compartilhamento de informações, a troca mútua de aprendizado e a garantia de um atendimento integral aos usuários do SUS. Assim, a EPS, à luz de um referencial teórico-metodológico problematizador, torna-se uma ferramenta imprescindível na formação continuada de futuros profissionais da saúde ${ }^{(8)}$.

Atualmente, o SUS vivencia uma mudança nas demandas sanitárias apresentadas pela população, traduzida pelas transições demográfica, nutricional e epidemiológica. Frente a essa mudança de paradigma, o programa de RMS é apresentado como estratégia de reorientação da atenção primária, sendo um contraponto ao modelo biomédico assistencial restritivo que outrora era hegemônico. Além de firmar-se em ações assistenciais holísticas, visto que a interdisciplinaridade assegura o princípio da integralidade ao agregar diferentes conhecimentos em saúde ${ }^{(9)}$.

A RIS traz a perspectiva de uma formação pautada na prática clínica e orientada pelos princípios do SUS, observando as necessidades e realidades loco-regionais. Imputa-se ao residente o perfil de liderança científico-política na qualificação da linha de cuidado na qual ele está inserido, visando a promoção, proteção e recuperação da saúde, embasando-se na colaboração interprofissional, na integralidade e intersetorialidade ${ }^{(10)}$.

A formação multiprofissional deve sempre nortear um trabalho em equipe, embasada na gestão, cooperação, parceria e respeito mútuo dos seus integrantes. Logo, o residente deve buscar junto aos colegas as melhores estratégias de atuação dentro dos serviços, utilizando-se de uma prática humanizada e imbuído de um olhar crítico-reflexivo (II).

\section{Modelo ativo problematizador na residência mul- tiprofissional}

Regulamentada pela Lei Federal $n^{\circ}$ II.I29 de 2005, em parceria do Ministério da Saúde (MS) com o Ministério da Educação, os programas de residências multiprofissionais visam a formação de profissionais dialogantes entre si e com as necessidades práticas por eles vivenciadas. Para isso, as RMS são construídas sobre planos pedagógicos centradas na EPS, no protagonismo estudantil, e no modelo de formação ativa ${ }^{(12)}$.

Projetos pedagógicos ativos devem ainda fornecer substrato ao residente para o reconhecimento das dimensões culturais, subjetivas e sociais do paciente, uma vez que incorporam ao seu escopo o conceito de problematização em saúde, imprescindível na formação integral do profissional. Dimensões estas até então alheias aos olhos dos profissionais formados pela modelo biomédico tradicional ${ }^{(13)}$.

A problematização em saúde cumpre seu papel formador ao fomentar nos estudantes o desejo de solucionar problemas práticos observados a partir da realidade. Dessa maneira, eles não só consolidariam novos conhecimentos, mas também exercitariam a autonomia por meio do seu protagonismo no processo de aprendizagem ${ }^{(13)}$.

\section{Perspectivas dos preceptores na implementação do plano pedagógico da RMS}

Os preceptores são profissionais da equipe qualificados e designados pela área de ênfase para atuarem como suporte aos residentes, permitindo o trabalho entre os diferentes integrantes da equipe multiprofissional, além de serem ponte para a população por ela assistida ${ }^{(14)}$.

Para alcançar as competências definidas no perfil pro- 
fissional do preceptor, é fundamental que este conheça o que está contemplado no PPP da RMS, acerca da atuação em equipe multiprofissional, promovendo a integração entre os conhecimentos, experiências e práticas das diversas categorias envolvidas no cuidado em saúde ${ }^{(12)}$.

Neste aspecto, quando indagados sobre o conhecimento do PPP da RMS e a situação em que obtiveram este conhecimento, os preceptores afirmaram que sua participação se fez desde o projeto de criação da residência no hospital em estudo, assim como a realização de cursos em preceptoria, que definem como fundamental para o conhecimento acerca do projeto pedagógico e o discernimento dos objetivos e diretrizes propostos pela instituição na formação dos residentes.

\section{Formação interprofissional em saúde, nuances e peculiaridades}

São discutidas desde o final da década de 1980 propostas de mudanças na formação em saúde no Brasil. Entretanto, esta discussão ganhou notoriedade quando o MS assumiu a responsabilidade de orientar e coordenar a formação dos profissionais da saúde para atender as necessidades exigidas pelo SUS ${ }^{(15)}$.

É necessário um diálogo permanente entre o mundo acadêmico e o mercado de trabalho, redesenhando um novo significado para as práticas em saúde ao se conectar com a teoria, definindo ações e atributos profissionais. As práticas de formação desenvolvidas nos espaços das residências requerem metodologias que promovam a discussão para a reflexão acerca do trabalho em saúde nas múltiplas esferas sociais ${ }^{(16)}$.

Neste processo, destacam-se as rodas de conversa como espaços de EPS, que viabilizam reflexões acerca das necessidades de saúde, o planejamento e pactuação dos processos de organização de serviço. As metodologias variadas estão de acordo com o projeto pedagógico da RMS, que trata de cada uma e deixa claro como são desenvolvidas. Os sujeitos são tomados como atores reflexivos da prática e construtores de conhecimento e alternativas de ações ${ }^{(17)}$.

"Tendo em vista as rodas de núcleo e de campo [reuniões para o debate, estudo e reflexão crítica], supervisões e pelos práticos cenários vivenciados, envolvendo preceptores e residentes" (Depoimento de Preceptor).

"No cotidiano dos residentes e nas reuniões para elaboração de estratégias de atuação, através das rodas de núcleo" (Depoimento de Preceptor).

A EPS, considerada alicerce para a prática multiprofissional, é determinada por uma gama de fatores, como valores interpessoais, articulação de saberes e o desenvolvimento de competências em grupo. $\mathrm{E}$ está margeada nas relações entre os indivíduos integrantes do programa de residência, almejando a atuação harmoniosa e o esforço conjunto ${ }^{(12)}$.

A roda de núcleo é um espaço destinado ao diálogo profissional e facilitada pelo preceptor e com a participação dos residentes da categoria. Constitui um espaço local de discussão do processo de trabalho, dos temas e situações complexas do dia-a-dia.

A tenda invertida se desenvolve em serviço, onde o preceptor de cada categoria acompanha o residente nos cenários de práticas, desde o ambulatório à visita clínica em leito, facilitando a integração do residente com os demais profissionais e orientando ações por ele desenvolvidas.

"Penso que houve melhoras nos últimos anos e as RMS tiveram um destaque essencial” (Depoimento de Preceptor).

"No dia a dia dos residentes e nas reuniões para criação das maneiras e estratégias de atuação" (Depoimento de Preceptor).

Apesar da ausência do termo "tenda invertida" na fala dos entrevistados, foi possível capturar seu sentido e sua utilização nos depoimentos.

O modo de "pensar" e "fazer" saúde, nesse contexto, implicariam em uma nova lógica e organização do trabalho, demandando um processo educacional que possibilite aos gestores e trabalhadores do sistema de saúde $\circ$ uso de outros conhecimentos, métodos, saberes e formas de atuação em equipe. Desta forma, a EPS deve ser favorecida no cotidiano de trabalho por meio das reflexões coletivas acerca das vivências e dos problemas identificados, contribuindo para a formação dos profissionais e, consequentemente, para a concretização dos princípios do SUS ${ }^{(12)}$.

\section{Práticas integrativas de formação no meio hos- pitalar e estratégias para multiprofissionalidade}

A busca por estratégias modificadoras de práticas e conceitos biomédicos tem revelado a necessidade do SUS adotar novos métodos de aprendizagem, incorporando práticas formativas críticas para sua manutenção. Neste sentido, a EPS aponta modelos inovadores em gestão participativa, aproximando o SUS de uma atenção integral à saúde ${ }^{(9)}$.

"EPS prioriza estratégias voltadas para Educação Permanente. Nos prende no serviço, e todos se envolvem no trabalho interdisciplinar [...]" (Depoimento de Residente).

Compreende-se como a educação permanente é aplicada em loco, observando o discurso do participante acima.

"[...] os módulos são fundamentais nas intervenções e reflexões sobre a conduta ética em saúde, vivenciada pelos residentes [...] A prática é imprescindível para a formação do mesmo. Ela 
guiará e permitirá aquisição de enriquecedoras experiências[...]" (Depoimento de Residente).

Nesta fala é verificado que além do conhecimento teórico, torna-se essencial a prática para esse sujeito, ressaltado experiências que para ele são engrandecedoras no processo de aquisição de conhecimentos.

Durante a formulação do SUS, destacamos avanços no campo da saúde, com ênfase na atenção pública. Todavia, identificam-se questões que demandam novas respostas frente aos desafios sanitários, desde o aprimoramento do sistema até reformulações nas práticas formativas, assistencialistas e de diálogo interprofissional.

"A teoria no âmbito hospitalar necessita de reformulação [...] práticas aplicadas deixam a desejar na formação do residente, sobretudo nas discussões em grupo, que devem caminham para a realidade comunitária [...]" (Depoimento de Residente).

É possível destacar nesta fala as diferenças entre os conteúdos aplicados e a real necessidade da comunidade. Observando que muitas estratégias tomadas por unidades formadoras se tornam incoerentes diante das demandas apresentadas aos trabalhadores pelo território aos quais pertencem.

É necessário portanto a elaboração e adoção de estratégias de gestão do trabalho em saúde, contribuindo para a articulação e coesão da equipe e para a soma dos conhecimentos, tendo em mente o ser humano como algo complexo no processos de saúde-doença( ${ }^{(9,11)}$.

A integralidade requer a implementação clara e precisa de uma formação comprometida com competências gerais, compatíveis com práticas afins a todos os profissionais de saúde envolvidos, unindo o conhecimento teórico à sua aplicabilidade no cenário prático ${ }^{(16)}$.

"[...] precisamos trabalhar unidos ou distantes nos cuidados ao doente [...] a quantidade de profissionais envolvidos depende das necessidades apresentadas pelo enfermo. Penso que esteja em sintonia com as metas e objetivos da residência e especialização interprofissional" (Depoimento de Residente).

A multiprofissionalidade prediz uma maior efetividade do cuidado, gerada por meio de articulação dos diferentes conhecimentos profissionais. $O$ que é deixado explícito pelo sujeito no seu discurso.

"Logo no início nos estimulam ao trabalho interprofissional. Atuando em grupos de várias especialidades. E almejando um olhar multiprofissional com a criação de um plano terapêutico único. Um olhar em grupo é o objetivo essencial do trabalho multiprofissional" (Depoimento de Residente).

Segundo o depoimento acima, desde o primeiro ano os residentes são estimulados a trabalhar de forma multiprofissional, valorizando este método e a adoção de projetos práticos.

A EPS se fundamenta na assimilação ativa do saber e na reflexão de práticas dentro dos serviços e, consequentemente, na problematização da realidade que os cerca. Ela busca a construção e reconstrução dos saberes, caracterizando-se pelas vivências diárias dos profissionais, os quais, por meio da problematização, são levados a uma reflexão crítica e na tomada de ações benéficas e ágeis a tais necessidades ${ }^{(12)}$.

É inquestionável que as práticas dos profissionais de saúde devem responder às demandas do SUS, com o trabalho interdisciplinar entre as diferentes categorias, como identificado no depoimento seguinte:

"É verídico o esforço multiprofissional, porém, vejo a existência de pontos vulneráveis na ação de vários profissionais da saúde [...] A EPS objetiva um trabalho orquestrado por toda equipe, 0 que se faz distante do nosso dia a dia" (Depoimento de Residente).

A RMS se caracteriza por permitir espaços de diálogo sobre educação permanente, incrementando competências necessárias aos profissionais de saúde para atuação em diferentes cenários apresentados no SUS.

\section{Estratégias e desafios para implantação da RMS - Da teoria à aplicabilidade prática}

A implantação da residência interprofissional trouxe uma maior aplicação prática do conhecimento acerca do território de atuação. Enalteceu ações em saúde coletiva, acordando com o princípio da integralidade da atenção, priorizando uma visão completa das pessoas e da comunidade a elas pertencentes, no que tange suas diversas nuances. Julga-se ainda a necessidade de uma maior articulação coletiva de ambos os envolvidos neste processo formativo, para que a proposta da residência se consolide como caminho para a mudança no paradigma de formação biomédica ainda em voga ${ }^{(9)}$.

É objeto deste programa de residência a implantação da Educação Permanente em Saúde, de acordo com seus documentos oficiais. Apesar dos percalços impostos durante sua implantação e execução, ele se mostra essencial para as redes de saúde do Ceará. É enfática a necessidade de transposição dos desafios apresentados, porém, é válido destacar os avanços obtidos nessa área, considerando a formação e o trabalho em saúde.

Ao serem interrogados sobre a implantação da RMS no hospital, os preceptores destacaram os seguintes pontos:

\footnotetext{
"Vivemos quatros anos compartilhando conhecimentos e reflexões" (Depoimento de Preceptor).
} 
"A RMS transformou vários serviços, contribuindo para multiprofissionalidade, e com a valorização dos outros colegas da saúde. Bem como suscitou a integralidade da assistência ao doente. Foram criados diversos serviços dentro da instituição a partir de demandas oriundas da residência multiprofissional [...]" (Depoimento de Preceptor).

"Foi espetacular, juntaram-se vários profissionais antes distante e muitas vezes sem relacionamento em um mesmo ambiente" (Depoimento de Preceptor).

Os preceptores consideraram a RMS como um método inovador, promotor da participação de diferentes categorias profissionais, bem como ações estratégicas da prática multiprofissional em saúde.

Com os relatos sobre a implantação da RMS no hospital, assume-se que a RMS-ESP/CE possui em seu alicerce uma base comunitária, com ações e estratégias dirigidas às reais necessidades por elas apresentadas. Deste modo, são cruzadas as barreiras institucionais ao incitar a territorialização e a criação de práticas promotoras dentro do território de responsabilidade da equipe.

O compromisso com uma assistência integral à saúde se dá por meio de reflexões acerca da prática cotidiana e de modalidades ativas de aprendizagem, algo fundamental para a efetivação de estratégias dirigidas a problemas reais do dia a dia ${ }^{(8)}$.

"A presença dos residentes engrandeceu muito o HM. Conseguimos ampliar o atendimento aos diferentes setores" (Depoimento de Preceptor). "Diversos serviços institucionais foram criados e vários profissionais egressos do programa de RMS foram contratados, corroborando para a importância de um plano pedagógico bem estruturado" (Depoimento de Preceptor).

Analisar o depoimento de trabalhadores no tocante às carências de saúde que entremeiam o cotidiano do serviço contribui para o reforço da gestão e da política institucional, instruindo medidas de aprimoramento profissional, melhorando a performance do atendimento e minimizando riscos no atendimento à população(18).

Apesar de serem pilares dogmáticos do SUS, a equidade e a universalidade são rotineiramente postos em cheque dentro dos serviços públicos de saúde, como observado nos seguintes relatos:
"Anseios maiores estão ligados à falta de preceptores que melhor orientem as necessidades apresentadas pelos residentes" (Depoimento de Preceptor).

"[...] falta de reconhecimento também corrobora para o desgaste profissional" (Depoimento de Preceptor).

É possível extrair do discurso acima uma percepção negativista do trabalho em serviço público de saúde, caracterizado pela falta de reconhecimento e valorização profissional.

Foi promulgada através da Portaria número I.I I I, de 5 de Julho de $2005^{(19)}$, bolsas de auxílio financeiro aos residentes, com o intuito de sedimentar e expandir as residências no País, recurso este financiado através de meios públicos, via MS, possibilitando uma dedicação exclusiva dos profissionais envolvidos, condição fundamental para um trabalho centrado e objetivado, que é exigido neste método formativo.

Assim, o objetivo da integralidade torna-se concreto, junto ao fortalecimento de representações dos segmentos de gestão, atenção, ensino e controle social em saúde.

\section{CONCLUSÃO}

Ao final da análise pode-se afirmar que, na perspectiva dos residentes e preceptores, o processo de formação proporcionado pela residência é fator fundamental na relação entre ensino-serviço com base nas competências e habilidades já adquiridas por eles através da sua formação.

Nesse processo, são sujeitos efetivos nas mudanças perante a realidade em que estão inseridos, contribuindo para novas estratégias na resolução de problemas, criação de novos projetos e elaboração de instrumentos de trabalho que são desenvolvidas durante o percurso na residência.

Nesta perspectiva, para que seja alcançada a integralidade das ações, é imperativo que o trabalho seja desenvolvido em equipe interprofissional, por meio do diálogo constante, favorecendo assim, a união e a troca de saberes e fazeres entre os profissionais da saúde.

Ao compreender a integralidade em saúde baseado na intra-setorialidade, intersetorialidade e na interdisciplinaridade, que são aspectos essenciais na formação no âmbito das residências, sugere-se que outros estudos sejam realizados com foco no processo de formação das residências multiprofissionais de base comunitária. 


\section{REFERÊNCIAS}

I.Vieira SP, Pieranton CR, Magnago C, Ney MS, Miranda RG.A graduação em medicina no Brasil ante os desafios da formação para a Atenção Primária à Saúde. Revista Saúde Debate [Internet]. 2018 [acesso em 5 abr. 2020]; 42(I): I89-207. Disponível em: https://www.scielosp.org/article/sdeb/2018. v42nspe I//89-207/pt.

2. Oliveira MPR, Menezes IHCF, Sousa LM, Peixoto MRG. Formação e Qualificação de Profissionais de Saúde: Fatores associados à Qualidade da atenção Primária. Revista Brasileira de Educação Médica [Internet]. 2016 [acesso em 5 abr. 2020]; 40(4):547-559. Disponível em: http://www.scielo.br/scielo.php?script=sci_abstract\&pi$\mathrm{d}=$ SO I 00-550220 I 6000400547\&lng=en\&nrm=iso\&tlng=pt.

3. Silva CA, Araújo MD. Programa de Residência Multiprofissional em Saúde: o que mostram as publicações. Revista Saúde debate [Internet]. 2019 [acesso em 5 abr. 2020]; 43(I23): I240-1258. Disponível em: https://www.scielosp. org/article/sdeb/20 I9.v43n I 23/I240-1 258.

4. Emmi DT, Silva DMC, Barroso RF. Experiência do ensino integrado ao serviço para formação em Saúde: percepção de alunos e egressos de Odontologia. Revista Comunicação Saúde Educação [Internet]. 2018 [acesso em 5 abr. 2020]; 22(64):223-236. Disponível em: https://www.scielosp.org/ article/icse/2018.v22n64/223-236/pt.

5. Silva CT, Terra MG, Kruse MHL, Camponogara S, Xavier MS. Residência multiprofissional como espaço intercessor para a educação permanente em saúde. Revista Texto Contexto Enfermagem [acesso em 5 de abr. 2020]; 25(I):I-9. Disponível em: https://www.lume.ufrgs.br/ bitstream/handle/ I0 I83//4I52 I/000989/35.pdf?sequence $=$ I \&isAllowed $=y$.

6. Minayo MCS. $O$ desafio do conhecimento: Pesquisa qualitativa em saúde. $14^{\text {a }}$ edição revista e aprimorada. São Paulo: Hucitec; 2014.

7. Ministério da Saúde. Resolução $N^{\circ} 466$, de 12 de dezembro de 2012 [acesso em 10 abr. 2020]. Disponível em: https://bvsms.saude.gov.br/bvs/saudelegis/cns/2013/ res0466_12_12_2012.html.

8. Filho JRF, Silva CBG, Costa MV, Forster AC. Educação Interprofissional nas políticas de reorientação da formação profissional em saúde no Brasil. Revista Saúde Debate [Internet]. 2019 [acesso em 5 abr. 2020]; 43 (1):86-96. Disponível em: https://www.scielosp.org/article/sdeb/2019. v43nspel/86-96/pt.

9. Almeida RGS, Teston EF, Medeiros AA. A interface entre o PET-Saúde/ Interprofissionalidade e a Política Nacional de Educação Permanente em Saúde. Revista Saúde Debate [Internet]. 2019 [acesso em 5 abr. 2020]; 43 (I):97-105. Disponível em: https://www.scielosp.org/article/sdeb/2019. v43nspel/97-105.

10. Júnior NC, Teston EF, Montanari PM, Ávila LK.Apresentação - Educação interprofissional em saúde na integração ensi- no e trabalho: apontamentos e contribuições da professora Regina Marsiglia para esse campo. Revista Saúde Soc. [Internet]. 2018 [acesso em 5 abr. 2020]; 37 (4):976-979. Disponível em: https://www.scielosp.org/article/sausoc/2018. v27n4/976-979/pt.

I I. Correio NGM, Correio DAM.A formação multiprofissional em saúde sob a ótica do residente. Revista Online de Pesquisa [Internet]. 2018 [acesso em 5 abr. 2020]; 10 (2):593598. Disponível em: https://search.proquest.com/openview/5add9fce88d I f632e3f I 7309ce055f03/ I ?pq=-origsitegscholar\&cbl=2030I83.

12. Mello AL, Arruda GT, Terra MG, Amemann CT. Fatores que interferem no ensino e aprendizagem de residentes multiprofissionais em saúde: revisão integrativa. Revista Arquivos Brasileiros de Ciências da Saúde [Internet]. 2019 [acesso em 5 abr. 2020]; 44 (2):।38-|46. Disponível em: https://nepas.emnuvens.com.br/abcshs/article/ view/I 176.

13. Silva AN, Senna MAA, Teixeira ACB, Lucietto DA, Andrade IA. $O$ uso de metodologia ativa no campo das Ciências Sociais em Saúde: relato de experiência de produção audiovisual por estudantes. Revista Interface comunidade, saúde, educação [Internet]. 2019 [acesso em 5 abr. 2020]; 24:I - I4. Disponível em: https://www.scielosp.org/article/icse/2020. v24/el9023I.

14. Souza SV, Ferreira BJ. Preceptoria: perspectivas e desafios na Residência Multiprofissional em Saúde. Revista Arquivos Brasileiros de Ciência da Saúde [Internet]. 2019 [acesso em 5 abr. 2020]; 44 (I):I 5-2I. Disponível em: https://www. portalnepas.org.br/abcshs/article/view/l 074.

15. Silva RMB, Moreira SNT. Estresse e Residência Multiprofissional em Saúde: Compreendendo Significados no Processo de Formação. Revista Saúde Debate [Internet]. 2019 [acesso em 5 abr 2020]; 43 (4):I57166. Disponível em: http://www.scielo.br/scielo.php?pid=SO I00-550220 I 90004001 57\&script=sci_arttext.

16. Alves MJ, Araújo HPA, Costa LAS, Marques MCS, Alencar RA. Ação Interdisciplinar de promoção à saúde no programa escola da família: relato de experiência de residentes do programa multidisciplinar em saúde da família. Revista Nursing [Internet]. 2019 [acesso em 5 abr. 2020]; 22 (252):2875-2877. Disponível em: https://pesquisa.bvsalud. org/portal/resource/pt/biblio-998974.

17. Lorena SB, Andrade MM, Arcoverde AMH, Vilela LS, Mota LRA, Sobrinho JEL. Análise do Acesso à Informação Acadêmica entre Estudantes de Medicina Inseridos numa Metodologia Ativa de Aprendizagem. Revista Saúde Debate [Internet]. 2019 [acesso em 5 abr 2020]; 43 (4): I76-186. Disponível em: http://www.scielo.br/ scielo.php?pid=S0 I 00-550220 19000400 | 76\&script=sci_arttext.

18. Nascimento DMM. Mediação de conflitos na gestão da saúde (médica, clínica e hospitalar): humanização do direito 
médico. Revista Cadernso Ibero-Americanos de Direit Sanitário [Internet]. 2020 [acesso em 5 abr. 2020]; 9 (I): I70195. Disponível em: https://www.cadernos.prodisa.fiocruz. br/index.php/cadernos/article/view/605.

1 9. Ministério da Saúde.Portaria N I. I I I, de 05 de julho de 2005 [acesso em 9 abr. 2020]. Disponível em: http://bvsms.saude. gov.br/bvs/saudelegis/gm/2005/prt I I I I_05_07_2005.html.

Recebido: 2020-05-12

Aceito: 2020-10-29 